\title{
An empirical investigation of the benefit of increasing the temporal resolution of task-evoked fMRI data with multi-band imaging
}

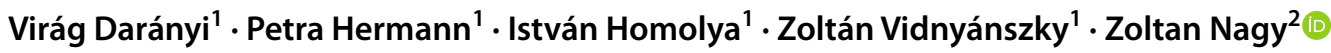

Received: 11 November 2020 / Revised: 26 February 2021 / Accepted: 3 March 2021 / Published online: 25 March 2021

(c) The Author(s) 2021

\begin{abstract}
Objective There is a tendency for reducing TR in MRI experiments with multi-band imaging. We empirically investigate its benefit for the group-level statistical outcome in task-evoked fMRI.

Methods Three visual fMRI data sets were collected from 17 healthy adult participants. Multi-band acquisition helped vary the TR (2000/1000/410 ms, respectively). Because these data sets capture different temporal aspects of the haemodynamic response (HRF), we tested several HRF models. We computed a composite descriptive statistic, $\mathrm{H}$, from $\beta$ 's of each firstlevel model fit and carried it to the group-level analysis. The number of activated voxels and the $t$ value of the group-level analysis as well as a goodness-of-fit measure were used as surrogate markers of data quality for comparison.

Results Increasing the temporal sampling rate did not provide a universal improvement in the group-level statistical outcome. Rather, both the voxel-wise and ROI-averaged group-level results varied widely with anatomical location, choice of HRF and the setting of the TR. Correspondingly, the goodness-of-fit of HRFs became worse with increasing the sampling frequency. Conclusion Rather than universally increasing the temporal sampling rate in cognitive fMRI experiments, these results advocate the performance of a pilot study for the specific ROIs of interest to identify the appropriate temporal sampling rate for the acquisition and the correspondingly suitable HRF for the analysis of the data.
\end{abstract}

Keywords Temporal sampling rate $\cdot$ fMRI $\cdot$ Goodness-of-fit $\cdot$ Multi-band $\cdot$ SNR

\section{Introduction}

After the development of in-plane parallel imaging methods in the 1990's, [1-3] slice-wise acceleration, a.k.a. simultaneous multi-slice (SMS) or multi-band (MB) imaging, was proposed by Larkman et al. in the early 2000's [4] and subsequently refined by others to become a widely utilized neuroimaging method [5-7].

A reasonable supposition is that increasing the temporal sampling rate (i.e. shorter repetition time (TR)) should improve the detection of blood-oxygen-level-dependent (BOLD) signal (see for example Feinberg et al. [8]). The benefit of higher number of data points per unit time is

Zoltan Nagy

zsydney@yahoo.com

1 Brain Imaging Centre, Research Centre for Natural Sciences, Budapest, Hungary

2 Laboratory for Social and Neural Systems Research, University of Zürich, Rämistrasse 100, P.O. Box 149, Zürich, Switzerland expected from the increased degrees of freedom for statistical analysis and the ability to more appropriately sample physiological processes (e.g. cardiac pulsations) up to around $1 \mathrm{~Hz}$.

Indeed, in resting-state functional magnetic resonance imaging (fMRI), MB imaging has shown utility $[8,9]$. However, it is still unclear when the benefits of the higher temporal sampling outweigh the correspondingly incurred SNR loss in task-based fMRI efforts [10-12]. The SNR loss is partly due to incomplete $\mathrm{T} 1$ relaxation during the short TR [13]. Larger MB acceleration factors often make use of in-plane parallel imaging acceleration as well, which imparts an additional spatially variable reduction in SNR through the $\mathrm{g}$-factor (although not the multi-band acceleration factor) [4, 14]. Furthermore, due to the shorter TR, temporal autocorrelations may also increase in the voxel-wise time course of fMRI data, which require careful additional processing before reliable statistical inference can be drawn $[15,16]$.

The physiological response to neural activity is highly variable across anatomical regions [17] as well as between participants or upon repeated scanning in a single participant 
[18]. The BOLD activation is usually ascertained by fitting one of several hemodynamic response functions (HRFs) to the fMRI time-series data (e.g. see chapter 14 in Friston et al. [19]). These models have a varying number of fitted variables, some of which capture different temporal aspects of the hemodynamic response. Therefore, an additional practical complexity is if and how much the additional data with the increased temporal sampling rate will impact the detection of the BOLD response while fitting these various models.

The overarching aim of this study was to empirically assess whether MB imaging data would provide a universal benefit regardless of anatomical area of the brain or the HRF used. In a realistic expectation, we hypothesized that such benefit, even if universally detectable, would likely be modulated by the anatomical location and/or the HRF model used. Therefore, we systematically tested five different HRF models in four different occipito-temporal regions of interest.

\section{Methods}

\section{Participants}

Each of the 21 healthy, right-handed human volunteers signed a written informed consent before undergoing a scanning session. Both the consent form and the scanning protocol were approved by the Health Registration and Training Centre (ENKK006641/2016/OTIG) and were in accordance with Helsinki Declaration of 1964 and its subsequent amendments. Four participants were excluded: one for excessive motion during the experiment, while three others had incomplete data. The remaining 17 participants (10 female/7 male) form the group described in this study. These data were utilized from a previous study with a separate aim that investigated the efficiency of MB sequences for different measurement durations [20].

\section{Data acquisition}

\section{Imaging protocol}

All magnetic resonance imaging (MRI) data were collected on a 3 T Siemens Magnetom Prisma scanner (Siemens Healthcare, Erlangen, Germany) and the vendor's $64 \mathrm{ch}$ receive-only head and neck coil (52 channels used for acquisition). Three different fMRI data sets were collected with 2D gradient echo echo-planar imaging (EPI) [21] with three different TRs. To increase the sampling rate of the BOLD signal (i.e. reduce TR), two of these acquisitions employed slice-wise multi-band acceleration of 4 or 6 [6]. Henceforth, the three fMRI sequences will be referred to as MB1 (i.e. without multi-band), MB4 and MB6, respectively.
In an attempt to keep the signal-to-noise ratio (SNR) optimum for each TR, the flip angle (FA) was set to the Ernst angle assuming a $\mathrm{T} 1$ relaxation time of $1200 \mathrm{~ms}$ for grey matter (GM). For a fair comparison, the total acquisition time, the echo time (TE) and the voxel dimensions were kept constant across the fMRI data sets (see Table 1 for the relevant image acquisition details). The MB4 and MB6 images were reconstructed as in Cauley et al. [22] to alleviate interslice signal leakage. The order of the three MB factors was pseudo randomized and balanced across the original of 21 volunteers. The randomization remained sufficient across the 17 participants included in the study (Table 1). In addition, the MB1 sequence was used for a short functional localizer experiment that identified four regions of interest (ROIs).

The imaging protocol also included a $1.0 \mathrm{~mm}$ isotropic resolution 3D T1-weigthed MPRAGE sequence [23] with $\mathrm{TR} / \mathrm{TE} / \mathrm{FA}=2300 \mathrm{~ms} / 3.03 \mathrm{~ms} / 9^{\circ}$ and inversion time of $900 \mathrm{~ms}$ for anatomical normalization.

\section{fMRI stimulus paradigm}

The above fMRI data were used to capture brain activity in response to a blocked event-related visual task paradigm (Fig. 1) [20]. Volunteers viewed grayscale images of faces, houses or headless bodies that were displayed centrally, subtending $3.8 \times 3.8^{\circ}$ on a uniform gray background. The visual stimuli were presented via an MRI-compatible LCD screen (32' NNL LCD Monitor, NordicNeuroLab, Bergen, Norway; refresh rate $60 \mathrm{~Hz}$ ) which was placed at $142 \mathrm{~cm}$ from the observer. For each 11-min fMRI data acquisition with one of the MB factors, stimuli were presented in five $\sim 1.5$-min blocks with $30 \mathrm{~s}$ rest before the first block and after the last block and $25 \mathrm{~s}$ rest periods between the blocks. The visual

Table 1 Sequence acquisition parameters and pseudorandomization of the order of MB factors

\begin{tabular}{llll}
\hline Parameter & MB1 & MB4 & MB6 \\
\hline TR (ms) & 2000 & 1000 & 410 \\
FA $\left(^{\circ}\right)$ & 79 & 64 & 45 \\
MB-factor & 1 & 4 & 6 \\
GRAPPA & 2 & N/A & N/A \\
Partial Fourier & N/A & $7 / 8$ & $7 / 8$ \\
Readout BW (Hz/pixel) & 2170 & 2170 & 2170 \\
Echo spacing (ms) & 0.55 & 0.57 & 0.61 \\
Image Volumes & 336 & 672 & 1638 \\
Time-series length & $11 \mathrm{~m} 20 \mathrm{~s}$ & $11 \mathrm{~m} 20 \mathrm{~s}$ & $11 \mathrm{~m} 17 \mathrm{~s}$ \\
TE (ms) & $30 \mathrm{~ms}$ & $30 \mathrm{~ms}$ & $30 \mathrm{~ms}$ \\
Voxel size & $3.0 \times 3.0 \times 3.0 \mathrm{~mm}^{3}$ & $3.0 \times 3.0 \times 3.0 \mathrm{~mm}^{3}$ & $3.0 \times 3.0 \times 3.0 \mathrm{~mm}^{3}$ \\
& $(25 \%$ slice gap) & $(25 \%$ slice gap) & $(25 \%$ slice gap) \\
Num of Slices & 36 & 36 & 36 \\
Scan order 1st & 6 & 5 & 6 \\
Scan order 2nd & 4 & 7 & 6 \\
Scan order 3rd & 7 & 5 & 5 \\
\hline
\end{tabular}




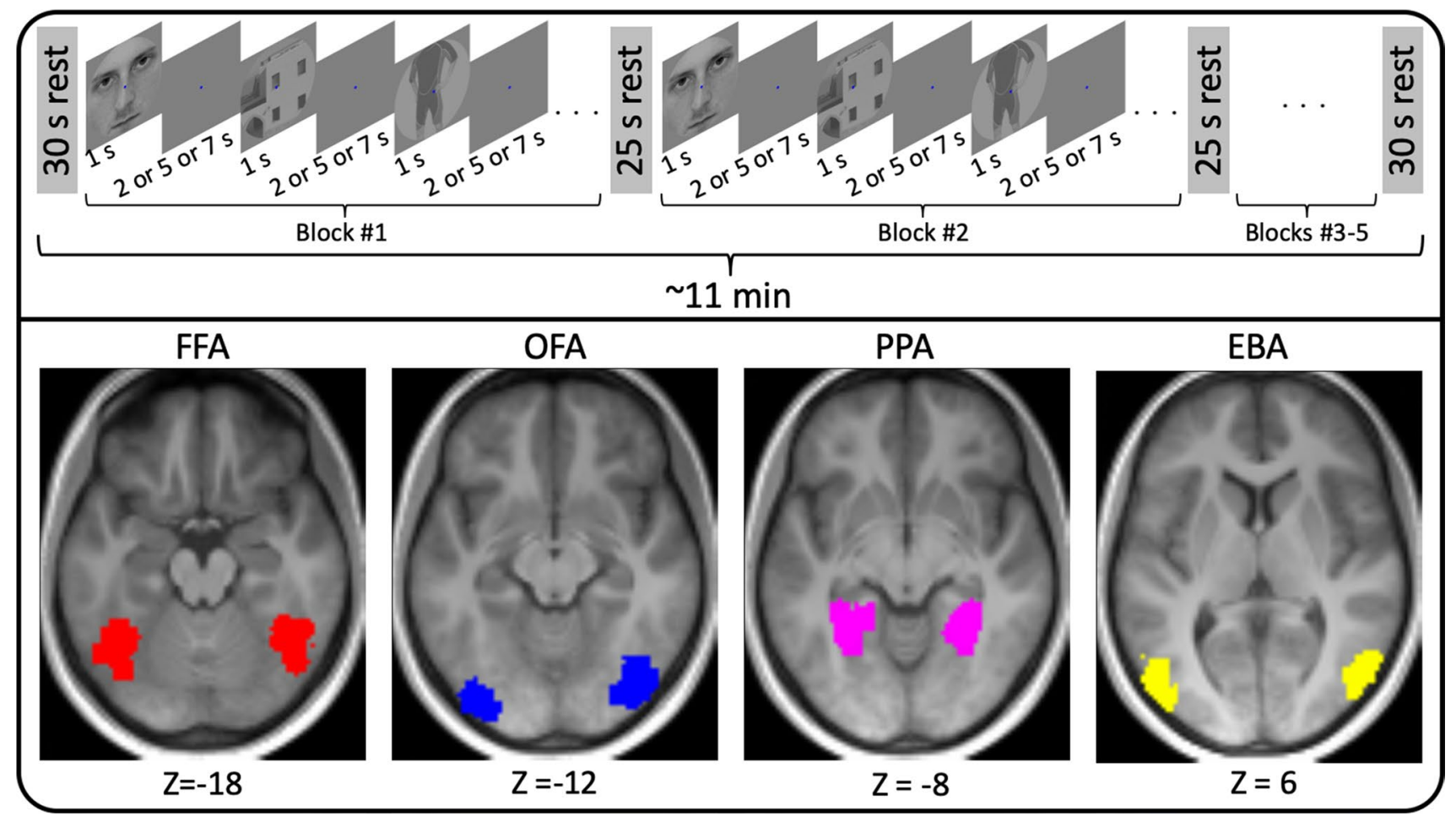

Fig. 1 Illustration of the visual stimulus paradigm (top) and depiction of ROI locations (bottom)

stimuli were separated by 2,5 or $7 \mathrm{~s}$ and then presented for $1 \mathrm{~s}$. Both the presentation order of the stimuli and the interstimulus interval were pseudo randomized in each of the blocks but the stimuli were identical for all participants and all three MB factors. The participants were instructed to fixate on a dot in the center of the visual display during the entire experiment. During the stimulus presentation, no response was requested from the volunteers. To ensure their attentiveness, after the fMRI data acquisition, they were presented with a group of photos and quizzed whether the photos were new or seen during the fMRI paradigm. Stimulus presentation was controlled by MATLAB R2015a (The MathWorks Inc., Natick, MA, USA) using PTB-3 (http:// psychtoolbox.org/credits).

\section{Data analysis}

All analyses were performed in Matlab (MathWorks, Natick, MA, USA) relying on the SPM12 software package [19] and custom-made Matlab scripts. Each of the three fMRI data sets with either MB1, MB4 and MB6 was fed through an identical preprocessing pipeline and subsequent statistical analysis steps with a general linear model (GLM) at the individual-level and each of the five different variants of the haemodynamic response function (HRF) in SPM. The final group-level effect was calculated via a random-effect design either voxel-wise or as an average within each of the four ROIs that were identified with the help of independent functional localizer scans (further details below).

\section{Pre-processing}

Before statistical analysis, each fMRI dataset was realigned to the first volume of each time-series, co-registered to the anatomical image of the same individual (via the mean of the realigned and unwarped time-series) and normalized to MNI152 space before being smoothed with a $6 \mathrm{~mm}$ FWHM 3D Gaussian kernel. As part of the GLM, high-pass filtering and pre-whitening were also implemented. Each data set was also segmented into tissue components and the GM segment was thresholded to include those above a probability of 0.1 [24].

\section{Haemodynamic response functions and effective $\beta$ 's}

Five commonly used, [19, 25, 26] built-in HRF models in SPM12 were independently fitted to the each of the three time-series (MB1/4/6). Namely, we employed

a. the canonical HRF model (CAN)

b. the canonical HRF model plus its temporal derivative (TD) 
c. the canonical HRF model plus both its temporal and dispersion derivatives (DD)

d. the finite impulse response model (FIR)

e. a combination of three gamma functions (GAM)

Importantly, these models provide varying flexibility by relying on different sets of basis functions that vary in number and shape. Furthermore, CAN is widely adopted in fMRI studies owing to its simple interpretability. Including temporal and dispersion derivatives enable the consideration of small variation in the timing and duration of the hemodynamic response, respectively. Finally, FIR and GAM are more flexible models for GLM fit with the former fitting to each time point from the onset of the stimulus a separate delta function.

Because these models differ in complexity and hence in the number of parameter estimates each of them yields through the GLM fit, direct comparison and interpretation are nontrivial. Therefore, for each of the models, we computed a single descriptive statistic, $H$, as an estimate of the HRF amplitude and used it in subsequent statistical analyses.

The CAN model yields a single parameter which is simply taken as $H$. Adding the derivative term to the CAN model will require one additional term but make the model more robust against slight time variation in BOLD response. Fitting both terms but using only the non-derivative term may induce amplitude bias due to the delay difference between the model and the measured time series. To counteract this effect, the estimated amplitude is calculated by incorporating the derivative term [27]. Therefore, for the TD model, we set

$H=\operatorname{sign}\left(\widehat{\beta}_{1}\right) \sqrt{\widehat{\beta}_{1}^{2}+\widehat{\beta}_{2}^{2}}$,

where $\widehat{\beta}_{1}$ is the estimated parameter for the canonical HRF and $\widehat{\beta}_{2}$ is for the temporal derivative term. Similarly, for DD, we set [28]

$H=\operatorname{sign}\left(\widehat{\beta}_{1}\right) \sqrt{\widehat{\beta}_{1}^{2}+\widehat{\beta}_{2}^{2}+\widehat{\beta}_{3}^{2}}$,

where $\hat{\beta}_{1}$ is the estimated parameter for the canonical HRF, while $\widehat{\beta}_{2}$ and $\widehat{\beta}_{3}$ are for the temporal and dispersion derivative terms, respectively. For the GAM model, we used the same approach and set

$H=\operatorname{sign}\left(\widehat{\beta}_{1}\right) \sqrt{\widehat{\beta}_{1}^{2}+\widehat{\beta}_{2}^{2}+\widehat{\beta}_{3}^{2}}$,

where $\widehat{\beta}_{1}, \widehat{\beta}_{2}$ and $\widehat{\beta}_{3}$ are the estimated parameters for the three fitted gamma functions. For FIR, we calculated the average of the estimated parameters assigned to the interval ranging between 4 and $6 \mathrm{~s}$ from the stimulus onset as
$H=\frac{1}{n} \sum_{i=t}^{t+n} \widehat{\beta}_{i}$

where $n$ is the number of averaged estimated parameters $\left(n=\operatorname{roundup}\left(\frac{2}{T R}\right)+1\right), t$ is the index of the first basis function which is at least $4 \mathrm{~s}$ from the stimulus onset and the subscript $i$ in $\widehat{\beta}_{i}$ counts the estimated parameters of the FIR model consecutively such that the $(t+n)$ th parameter is no more than $6.15 \mathrm{~s}$ from the stimulus onset.

The investigated interval for averaging was defined based on the individual HRF estimations using FIR, considering each subject and MB factor to include a period around the peak (Supplementary Fig. 1).

\section{Regions of interest}

Four category-selective visual cortical ROIs (Table 2), namely the fusiform face area (FFA), [29] occipital face area (OFA), [30] parahippocampal place area (PPA) [31] and extrastriate body area (EBA), [32] were defined individually based on anatomical landmarks and first-level statistical contrast maps (thresholded at $p<0.001$, uncorrected) of the independent functional localizer scans. The GLM for these statistical contrast maps relied on the canonical HRF. The ROIs were identified as responding to faces vs houses (FFA and OFA), houses vs faces (PPA) or bodies vs houses (EBA). The PPA and EBA were identified bilaterally in all participants, the FFA bilaterally in 16 participants, and OFA in 14 participants. For the ROI-based analyses, a sphere with a radius of $6 \mathrm{~mm}$ and a center located at the peak voxel of the identified ROI was defined for each subject.

Table 2 Peak voxel MNI coordinates for the four ROIs

\begin{tabular}{llrlll}
\hline ROI & Hemisphere & \multicolumn{1}{l}{$\mathrm{x}(\mathrm{mm})$} & $\mathrm{y}(\mathrm{mm})$ & $\mathrm{z}(\mathrm{mm})$ & $\mathrm{N}$ \\
\hline FFA & Right & $43 \pm 1.1$ & $-55 \pm 1.2$ & $-18 \pm 1.0$ & 16 \\
& Left & $-40 \pm 0.9$ & $-57 \pm 1.5$ & $-17 \pm 0.9$ & 16 \\
OFA & Right & $39 \pm 1.1$ & $-77 \pm 1.3$ & $-12 \pm 1.0$ & 14 \\
& Left & $-38 \pm 1.2$ & $-82 \pm 2.2$ & $-11 \pm 1.0$ & 14 \\
EBA & Right & $51 \pm 0.7$ & $-71 \pm 0.9$ & $2 \pm 1.2$ & 17 \\
& Left & $-48 \pm 1.0$ & $-75 \pm 1.6$ & $6 \pm 1.6$ & 17 \\
PPA & Right & $29 \pm 0.6$ & $-48 \pm 1.0$ & $-7 \pm 0.4$ & 17 \\
& Left & $-28 \pm 0.9$ & $-48 \pm 1.1$ & $-8 \pm 0.4$ & 17 \\
\hline
\end{tabular}

MNI coordinates ( $x, y, z$ in millimeters) are provided separately for both hemispheres and each investigated ROI. Provided data are mean \pm standard error across the number of participants $(N)$ for whom these regions were individually identifiable using $p<0.001$ uncorrected threshold

FFA fusiform face area, $O F A$ occipital face area, $P P A$ parahippocampal place area, $E B A$ extrastriate body area 


\section{Group-level effect}

Voxel-wise group-level random-effects analyses were performed on individual effect sizes of the variable $\mathrm{H}$ (i.e. HRF amplitudes resulted in the GLM fit in Eq. 1-4) for the main effect (i.e. face + house + body vs baseline) as well as separately for face, house and body for each multi-band factor and HRF model. The resulting $t$ value maps were thresholded at a false positive rate, $\alpha$, of $p<0.001$ (uncorrected) and multiplied by the GM mask.

Separate group-level effects were also obtained by calculating the average effect size, $\mathrm{H}$, within each of the ROIs (averaged across the hemispheres) for the contrast that is relevant for the ROI (face versus baseline for FFA and OFA, house versus baseline for PPA and body versus baseline for EBA). The results within the ROIs are based on averaging across the participants to compare HRFs as well as averaging across both subjects and HRFs to compare MB factors.

\section{ROI-based and voxel-wise ANOVA}

The main effect of MB acceleration and HRF model as well as their interaction were formally investigated with two separate analyses. In one of these analyses, the ROI average of the effect size, $H$, was extracted for each participant for all combinations of the three MB acceleration levels and the five HRF models and inserted into a two-way repeated-measures ANOVA in Matlab via the built in fitrm and ranova functions. Because 12 tests were made, the level of statistical significance was set at $0.05 / 12=0.0042$. The 12 tests were the main effects for MB acceleration, main effect for HRF model and their interaction in each of the 4 ROIs. The corresponding voxel-wise analysis was performed in SPM with MB acceleration and HRF model as within-subject factors. Finally, spm make contrast ( [ 3 5 $]$ ) provided the contrasts for the voxel-wise analyses of the main effects and the interaction term. Maps were thresholded at $p<0.001$, uncorrected.

\section{Additional outcome measures}

Apart from the group-level t statistics, we extracted the adjusted goodness-of-fit (GoF) coefficient $\left(R^{2}\right)$ for GLM fit with the help of the SPM MACS toolbox, [33] as well as the number of grey matter voxels exceeding the threshold for all group statistics. The former was used as a marker for the quality of the GLM fit for each MB factor and applied HRF model. These adjusted $R^{2}$ values were evaluated both voxel-wise and within the ROIs by averaging across the participants to compare HRFs or averaging across both subjects and HRFs to compare MB factors. The adjusted $R^{2}$ is an improved GoF measure that considers model complexity and

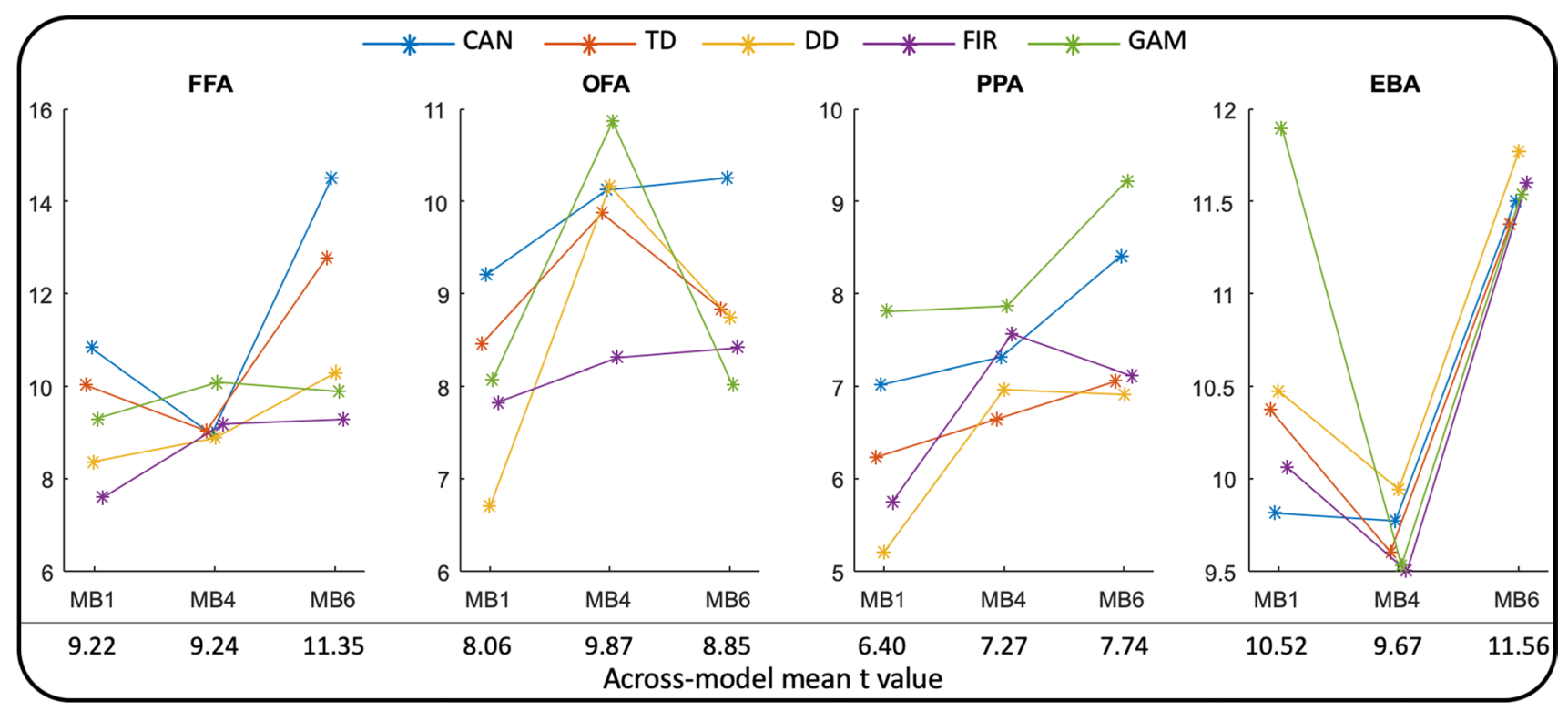

Fig. 2 ROI-averaged $t$ values ( $p<0.001$ uncorrected) from the grouplevel random-effects analyses. Four ROIs are shown from left to right for each of the three MB factors. For each data set in each ROI, five different HRFs were used for the analyses. For each of the MB factors, the corresponding $t$ values are averaged across HRF models and given below the plots. CAN Canonical HRF (blue), TD Canonical
$\mathrm{HRF}+$ its time derivative (red), $D D$ Canonical HRF + both its temporal and dispersion derivatives (yellow), FIR finite impulse response HRF (purple), GAM combination of three gamma functions (green), FFA fusiform face area, $O F A$ occipital face area, $P P A$ parahippocampal place area, $E B A$ extrastriate body area 


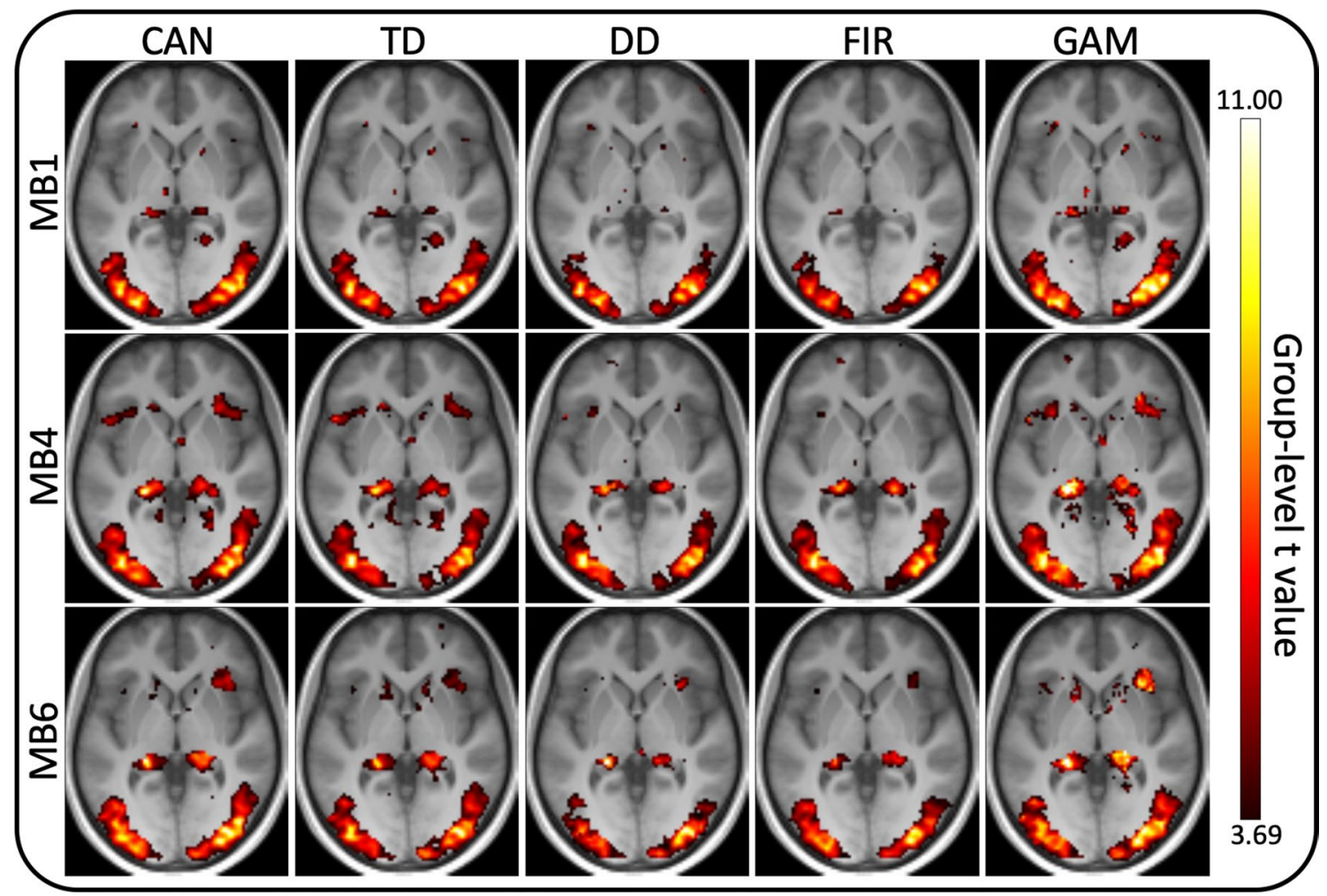

Fig. 3 Voxel-wise $t$ value ( $p<0.001$ uncorrected) maps from the group-level random-effects analyses, for each of three multi-band factors (rows) and five HRFs (columns). The background image is the axial slice at $z=0$ in MNI coordinate space. CAN Canonical HRF,

penalizes the fit in relation to the number of variables in the model [34].

\section{Results}

\section{Group-level ROI results}

The $t$ value for each ROI of the group-level statistical analysis was variable with respect to both anatomical location and HRF for each of the three MB factors but in all cases provided a robust activation with a mean $t$ value of at least 5 (Fig. 2). To assess whether the increased temporal sampling rate provides an overall benefit, the grouplevel $t$ values were further averaged across HRFs for an easier comparison of MB factors (listed below each subplot). Although in each ROI either MB4 or MB6 outperformed MB1 on average, the differences were sometimes minimal (e.g. MB1 vs MB4 in FFA), MB1 could be better (e.g. MB1 vs MB4 in EBA) and MB6 was not the overall winner.
$T D$ Canonical HRF + its time derivative, $D D$ Canonical $\mathrm{HRF}+$ both its temporal and dispersion derivatives, FIR finite impulse response HRF, GAM combination of three gamma functions

\section{Voxel-wise $t$ value maps}

Supporting the above results from the specific ROIs, voxelwise comparisons yielded similarly variable group-level $t$ values with respect to HRFs and MB factors (Fig. 3). When benefits of MB imaging can be identified, the MB acceleration factor need not be the highest achievable-in this case going from MB1 to MB4 often already provides all the benefit.

\section{ROI-based and voxel-wise ANOVA}

In summary, these analyses formalized and corroborated the above findings (Figs. 2, 3) in that main effect for HRF model is a consistent finding while the main effect of MB acceleration is only a weak predictor of group-level outcome. More specifically, in ROI analysis the main effect of the HRF model and the interaction of MB factor and HRF model were statistically significant at $p<0.0001$ for each of the four ROIs (Table 3 ). The 
Table 3 Results of the ROIlevel ANOVA

\begin{tabular}{lllll}
\hline & FFA & OFA & PPA & EBA \\
\hline MB & $F(2,30)=2.59$ & $F(2,26)=1.64$ & $F(2,32)=6.31$ & $F(2,32)=2.04$ \\
& $p=0.0915$ & $p=0.2137$ & $p=0.0049$ & $p=0.1468$ \\
HRF & $F(4,60)=24.97$ & $F(4,52)=14.78$ & $F(6,64)=21.46$ & $F(4,64)=41.15$ \\
& $p<0.0001$ & $p<0.0001$ & $p<0.0001$ & $p<0.0001$ \\
MBxHRF & $F(8,120)=15.82$ & $F(8,104)=13.11$ & $F(8,128)=16.97$ & $F(8,128)=34.75$ \\
& $p<0.0001$ & $p<0.0001$ & $p<0.0001$ & $p<0.0001$ \\
\hline
\end{tabular}

Both the $F$ value, degrees of freedom and the $p$ value are provided for the main effects of MB acceleration (top row) and HRF model type (middle row) as well as their interaction (bottom row) in each of the 4 ROIs (columns)

FFA fusiform face area, $O F A$ occipital face area, $P P A$ parahippocampal place area, $E B A$ extrastriate body area

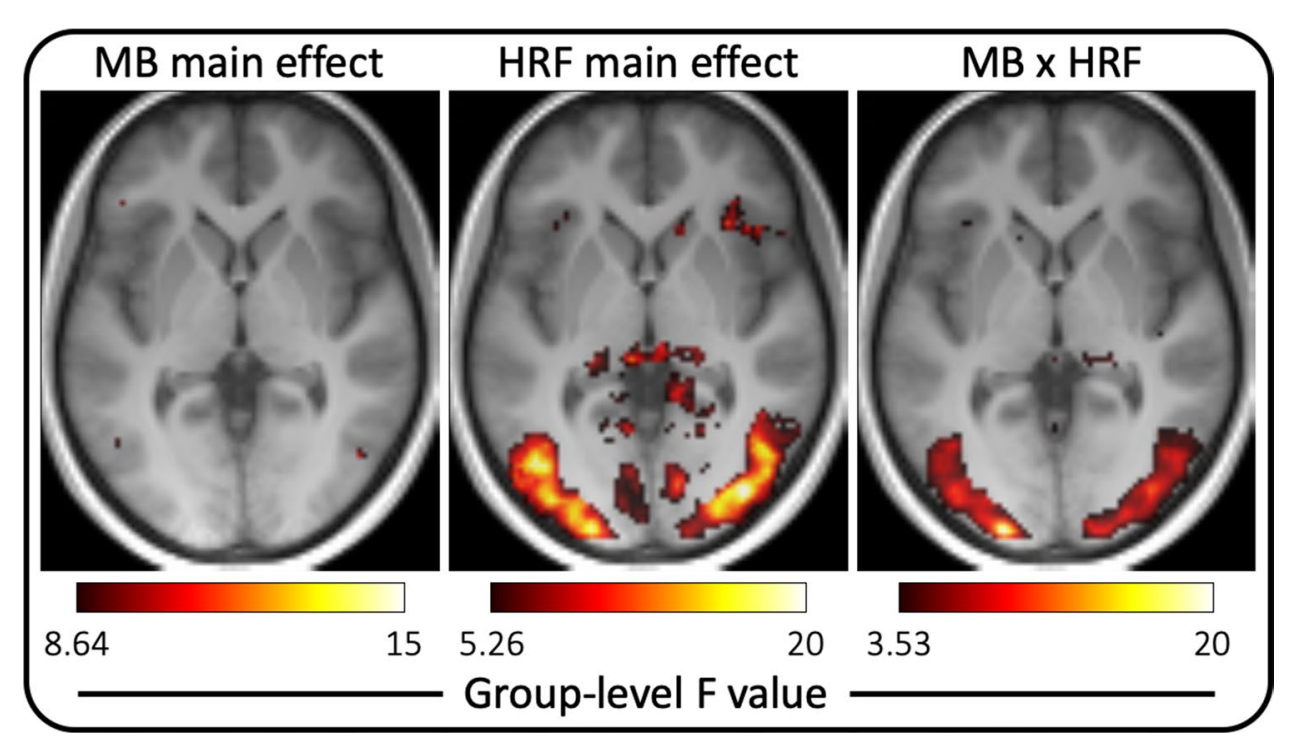

Fig. 4 Voxel-wise thresholded $F$ value $(p<0.001$ uncorrected) maps from the group-level two-way repeated-measures ANOVA with the main effect of MB acceleration factor (left, $F(2,32)=8.64)$, the main effect of the HRF model type (middle, $F(4,64)=5.26)$ and their interaction (right, $F(8,128)=3.53$ ). The background image is the axial slice at $z=0$ in MNI coordinate space. Note that the color scaling is different for the three subplots and were chosen in attempt to display the results without clipping or missing voxels main effect of MB factor was somewhat variable with $p=0.0915 / 0.2137 / 0.1468 / 0.0049$ in FFA, OFA, EBA and PPA, respectively. Note that without Bonferroni correction the main effect of MB acceleration would be statistically significant in the PPA. Nevertheless, the results of the corresponding voxel-wise SPM analysis (Fig. 4) indicated only a main effect for the HRF model type but not the MB acceleration factor, which suggests that the significant ROI-level finding without Bonferroni correction would likely be a false positive. A smaller effect for the interaction of these two variables could also be detected.

\section{Goodness of fit}

Figure 5 provides the adjusted goodness-of-fit values [33] averaged in each ROI and for each HRF for each of the three MB factors. Unsurprisingly, an almost universally monotonic decline is apparent as the MB factor is increased. Given the highly complex physiological mechanism of the BOLD response in comparison to the HRF models that contain only a few parameters, as more data are collected the more apparent the difference between the model and the data becomes. Voxel-wise results corroborate these conclusions both when averaged across participants or when averaged both across participants and across HRF models (Supplementary Fig. S2).

\section{Number of activated voxels}

Overall, MB imaging did not produce measurable difference in the number of activated voxels within the GM mask in the four contrasts that were tested (Fig. 6). Indeed, choice of the HRF imparted a larger effect on the number of activated voxels than did the MB acceleration factor. 


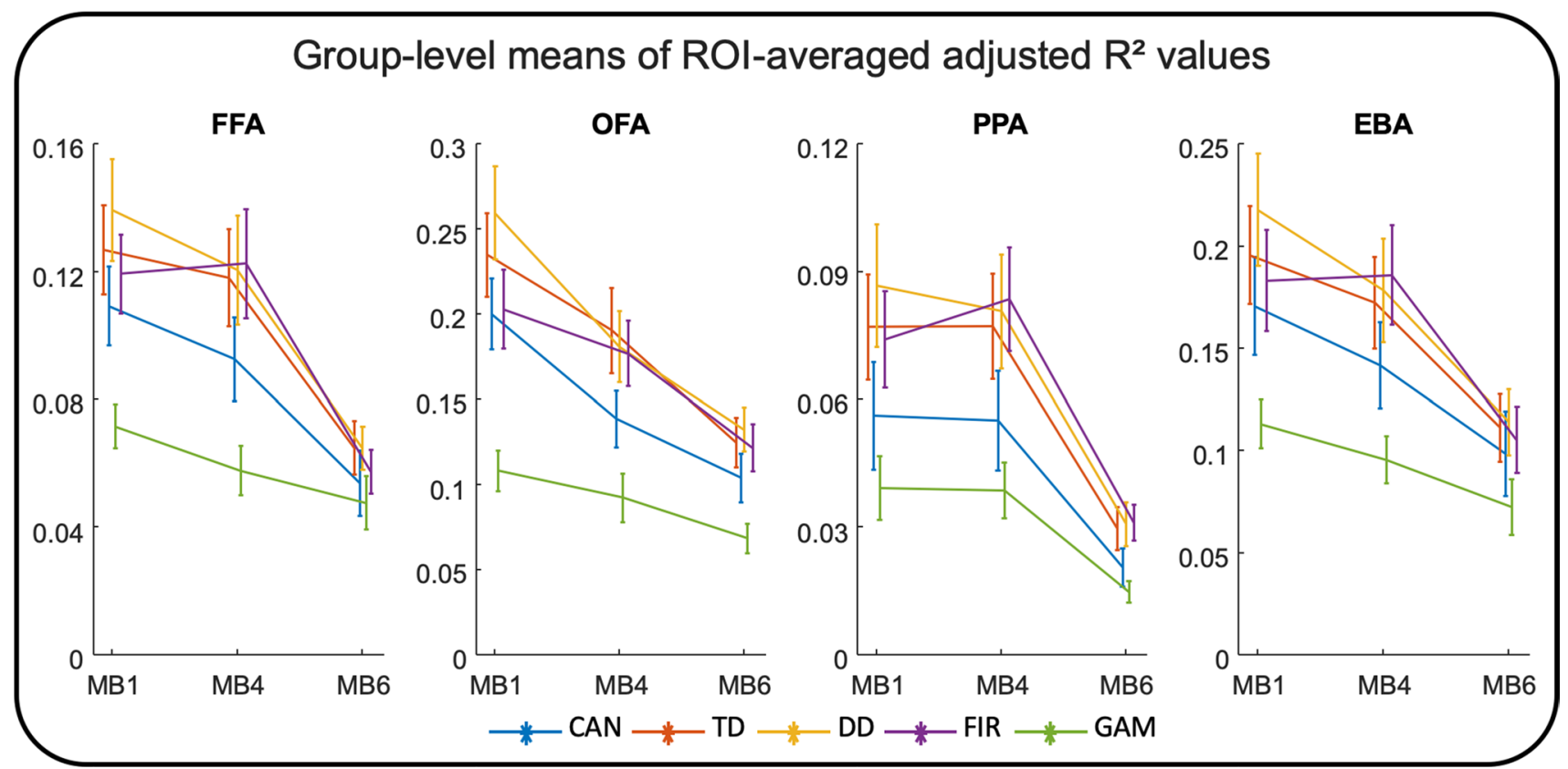

Fig. 5 Group-level mean of ROI-averaged adjusted $R^{2}$ values for each of three MB factors and five HRFs. As the MB factor is increased from 1 to 4 to 6 , the goodness-of-fit (i.e. adjusted $R^{2}$ ) values tend to drop in all observed ROIs. CAN Canonical HRF (blue), TD Canonical $\mathrm{HRF}+$ its time derivative (red), $D D$ Canonical HRF + both its tempo- ral and dispersion derivatives (yellow), FIR finite impulse response HRF (purple), GAM combination of three gamma functions (green), FFA fusiform face area, $O F A$ occipital face area, $P P A$ parahippocampal place area, $E B A$ extrastriate body area

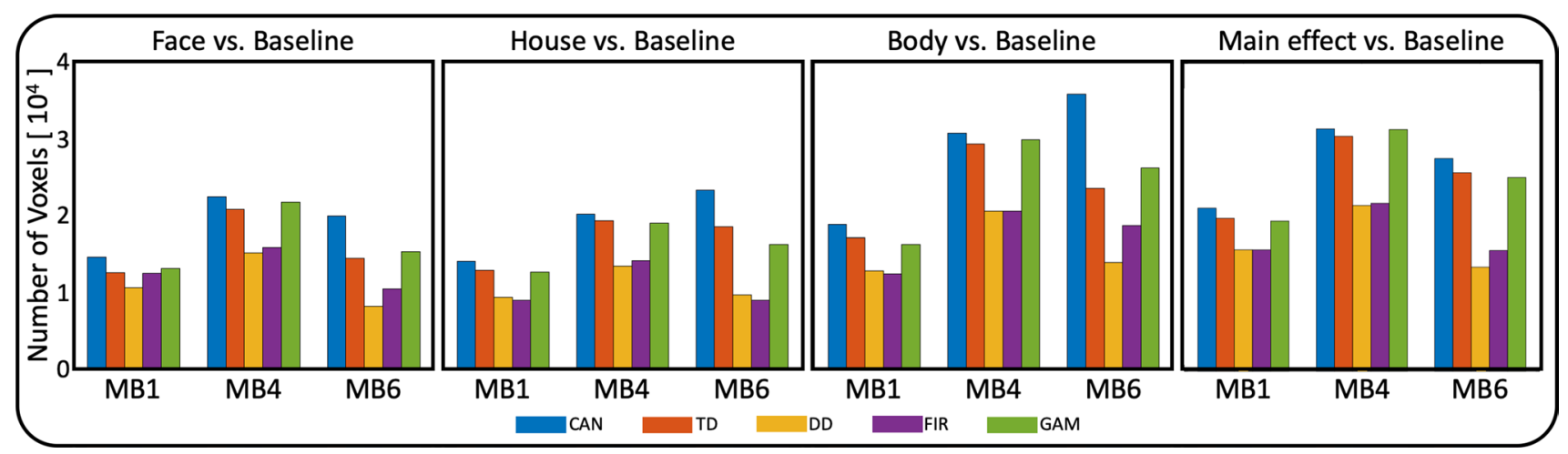

Fig. 6 Number of activated voxels ( $p<0.001$ uncorrected) after separate group-level random-effects analyses for face, house and body as well as their combined main effect while utilizing one of five different HRFs. The MB4 acceleration consistently results in the largest number of activated voxels. Notable however is that the choice of HRF can have a larger effect on the number of activated voxels (e.g. CAN on average outperforms DD by a larger margin). CAN Canonical HRF (blue), TD Canonical HRF + its time derivative (red), $D D$ Canonical HRF + both its temporal and dispersion derivatives (yellow), FIR finite impulse response HRF (purple), GAM combination of three gamma functions (green)

from increased temporal sampling of a given process, the practical limitations can balance out any such benefit. In the case of the nuclear magnetic resonance phenomenon, the sampling and signal generation processes are intricately intertwined through $\mathrm{T} 1$ relaxation, such that increased temporal sampling rate (i.e. shorter TR) results in lower SNR. Therefore, methods that reduce TR will often coincidentally reduce SNR as well. 
Lowering TR is achievable through various means. Although we utilized MB acceleration to maintain full coverage of the brain, it is imperative to state that the fluctuating benefit of the increased temporal sampling rate (e.g. see Fig. 2) is unlikely to be unique to MB imaging per se. For example, TR can be shortened by acquiring fewer slices in a multi-slice 2D EPI acquisition-imparting a similar SNR hit.

Previous output highlighted the fact that it is not always the higher MB acceleration factor that provides the best statistical outcome [35] and indicated a similar reduction of the mean $t$ value in subject specific ROIs and/or the number of activated voxels in a taskbased fMRI $[10,11]$. As these studies used different stimuli and/or different ROI(s), our results corroborate and widen the scope of their finding. Additionally, the explicit variation of HRF in the present work further supports the conclusion that the impact of temporal sampling can be dependent on several other acquisition or processing parameters.

We hypothesized that the choice of the HRF would impact the outcome of the group-level studies and modulate the effect of the MB factor, because the temporal evolution of the hemodynamic response varies with anatomical location in a given person $[17,36]$ as well as among individuals [18]. Therefore, fitting either HRF to the local time-series data will be imperfect to different degrees. Additional data, with higher temporal sampling rate, cannot be expected to impact each model identically.

The separate systematic evaluation of the adjusted GoF measure for each fit supports this notion (Fig. 4). Although, it is often taken for granted that additional data will improve results of regression, this should only be expected when the assumed model correctly captures all details of the process. Otherwise, the additional data will not improve the reliability of the fit and rather serve to pinpoint that the assumed model is not appropriate. Indeed, with additional data from MB4 the fit improves for some models in some anatomical areas (e.g. FIR in FFA) but not all, while the fit for data with MB6 universally worsened. Note that the adjusted GoF measure penalizes higher number of variables in a model, therefore even FIR (the most flexible model) does not improve with the additional data.

MB imaging can also be used to reduce total acquisition time. Although, this practice does not increase statistical degrees of freedom but the increased patient comfort may results in fewer and/or smaller motion artifacts. This aspect was not investigated here.

\section{Conclusions}

Increasing the temporal sampling rate of task-based fMRI data does not provide a universal benefit over the entire brain and outcome depends also on the choice of HRF in the statistical analysis pipeline. Given that all MB factors and HRFs provided robust activations, these results caution against the default use of acquisition methods with the highest possible sampling rate (i.e. lowest TR). Rather, a practically implementable recommendation is to perform a small pilot study with various TRs for the specific stimulus and cohort to identify the most appropriate settings for the full experiment involving the entire group of participants.

Supplementary Information The online version contains supplementary material available at https://doi.org/10.1007/s10334-021-00918-z.

Acknowledgements The authors gratefully acknowledge the input of Dr. Adam O. Kettinger in the form of fruitful discussion and practical guidance during the project.

Funding Open Access funding provided by Universität Zürich.

\section{Declarations}

Conflict of interest The authors have no conflict of interests to declare.

Ethical standards All procedures that involved the human participants were in accordance with the ethical standards of the institutional and/ or national research committee and with the 1964 Helsinki Declaration and its later amendments.

Informed consent Each participant signed a written informed consent form prior to the MRI scanning session.

Open Access This article is licensed under a Creative Commons Attribution 4.0 International License, which permits use, sharing, adaptation, distribution and reproduction in any medium or format, as long as you give appropriate credit to the original author(s) and the source, provide a link to the Creative Commons licence, and indicate if changes were made. The images or other third party material in this article are included in the article's Creative Commons licence, unless indicated otherwise in a credit line to the material. If material is not included in the article's Creative Commons licence and your intended use is not permitted by statutory regulation or exceeds the permitted use, you will need to obtain permission directly from the copyright holder. To view a copy of this licence, visit http://creativecommons.org/licenses/by/4.0/.

\section{References}

1. Pruessmann KP, Weiger M, Scheidegger MB, Boesiger P (1999) SENSE: sensitivity encoding for fast MRI. Magn Reson Med 42:952-962

2. Roemer PB, Edelstein WA, Hayes CE, Souza SP, Mueller OM (1990) The NMR phased array. Magn Reson Med 16:192-225 
3. Sodickson DK, Manning WJ (1997) Simultaneous acquisition of spatial harmonics (SMASH): fast imaging with radiofrequency coil arrays. Magn Reson Med 38:591-603

4. Larkman DJ et al (2001) Use of multicoil arrays for separation of signal from multiple slices simultaneously excited. J Magn Reson Imaging 13:313-317

5. Moeller S et al (2010) Multiband multislice GE-EPI at 7 tesla, with 16-fold acceleration using partial parallel imaging with application to high spatial and temporal whole-brain fMRI. Magn Reson Med 63:1144-1153

6. Setsompop K et al (2012) Blipped-controlled aliasing in parallel imaging for simultaneous multislice echo planar imaging with reduced g-factor penalty. Magn Reson Med 67:1210-1224

7. Feinberg DA, Setsompop K (2013) Ultra-fast MRI of the human brain with simultaneous multi-slice imaging. J Magn Reson 229:90-100

8. Feinberg DA et al (2010) Multiplexed echo planar imaging for sub-second whole brain FMRI and fast diffusion imaging. PLoS ONE 5:e15710

9. Smith SM et al (2013) Resting-state fMRI in the human connectome project. Neuroimage 80:144-168

10. Demetriou L et al (2018) A comprehensive evaluation of increasing temporal resolution with multiband-accelerated protocols and effects on statistical outcome measures in fMRI. Neuroimage 176:404-416

11. Chen L et al (2015) Evaluation of highly accelerated simultaneous multi-slice EPI for fMRI. Neuroimage 104:452-459

12. Todd $\mathrm{N}$ et al (2017) Functional sensitivity of $2 \mathrm{D}$ simultaneous multi-slice echo-planar imaging: effects of acceleration on g-factor and physiological noise. Front Neurosci 11:1-14

13. Haacke EM (1999) Magnetic resonance imaging: physical principles and sequence design. Wiley

14. Wald LL (2012) The future of acquisition speed, coverage, sensitivity, and resolution. Neuroimage 62:1221-1229

15. Corbin N, Todd N, Friston KJ, Callaghan MF (2018) Accurate modeling of temporal correlations in rapidly sampled fMRI time series. Hum Brain Mapp. https://doi.org/10.1002/hbm.24218

16. Sahib AK et al (2016) Effect of temporal resolution and serial autocorrelations in event-related functional MRI. Magn Reson Med 76:1805-1813

17. Nagy Z, Hutton C, Weiskopf N, Deichmann R (2008) Functional magnetic resonance imaging of the motor network with $65 \mathrm{~ms}$ time resolution. In: Proceedings of the 16th Annual Meeting of the International Society for Magnetic Resonance in Medicine 2519

18. Aguirre GK, Zarahn E, D'Esposito M (1998) The variability of human, BOLD hemodynamic responses. Neuroimage 8:360-369

19. Friston KJ, Ashburner JT, Kiebel SJ, Nichols TE, Penny WD (2007) Statistical parametric mapping: the analysis of functional brain images. Elsevier

20. Kiss M, Hermann P, Vidnyánszky Z, Gál V (2018) Reducing taskbased fMRI scanning time using simultaneous multislice echo planar imaging. Neuroradiology 60:293-302
21. Schmitt F et al (1998) Echo-planar imaging: theory technique and application. Springer

22. Cauley SF, Polimeni JR, Bhat H, Wald LL, Setsompop K (2014) Interslice leakage artifact reduction technique for simultaneous multislice acquisitions. Magn Reson Med 72:93-102

23. Mugler JP, Brookeman JR (1990) Three-dimensional magnetization-prepared rapid gradient-echo imaging (3D MP RAGE). Magn Reson Med 15:152-157

24. Ashburner J, Friston KJ (2005) Unified segmentation. Neuroimage 26:839-851

25. Glover GH (1999) Deconvolution of impulse response in eventrelated BOLD fMRI. Neuroimage 9:416-429

26. Ollinger JM, Shulman GL, Corbetta M (2001) Separating processes within a trial in event-related functional MRI. I. The method. Neuroimage 13:210-217

27. Calhoun VD, Stevens MC, Pearlson GD, Kiehl KA (2004) fMRI analysis with the general linear model: removal of latency-induced amplitude bias by incorporation of hemodynamic derivative terms. Neuroimage 22:252-257

28. Lindquist MA, Loh JM, Atlas LY, Wager TD (2009) Modeling the hemodynamic response function in fMRI: efficiency, bias and mis-modeling. Neuroimage 45:S187-S198

29. Kanwisher N, McDermott J, Chun MM (1997) The fusiform face area: a module in human extrastriate cortex specialized for face perception. J Neurosci 17:4302-4311

30. Pitcher D, Walsh V, Yovel G, Duchaine B (2007) TMS evidence for the involvement of the right occipital face area in early face processing. Curr Biol 17:1568-1573

31. Epstein R, Kanwisher N (1998) A cortical representation of the local visual environment. Nature 392:598-601

32. Downing P, Shuman Y, Kanwisher N (2001) A Cortical Area Selective for Visual Processing of the Human Body. Science (80-) 293:2470-2473

33. Soch J, Allefeld C (2018) MACS - a new SPM toolbox for model assessment, comparison and selection. J Neurosci Methods 306:19-31

34. Razavi M et al (2003) Model assessment and model building in fMRI. Hum Brain Mapp 20:227-238

35. Bhandari R et al (2020) Does higher sampling rate (multiband + SENSE) improve group statistics - an example from social neuroscience block design at 3T. Neuroimage 213:116731

36. Schacter DL, Buckner RL, Koutstaal W, Dale AM, Rosen BR (1997) Late onset of anterior prefrontal activity during true and false recognition: an event-related fMRI study. Neuroimage 6:259-269

Publisher's Note Springer Nature remains neutral with regard to jurisdictional claims in published maps and institutional affiliations. 